Case Report

Open Access

\title{
Idiopathic Polypoidal Choroidal Vasculopathy - A Case Report
}

\author{
Rahul Gupta $^{1 \star}$, Shairin Jahan ${ }^{1}$, Gargi Nagpal ${ }^{1}$, Naheed Akhtar ${ }^{2}$ and Abdul Waris ${ }^{2}$ \\ ${ }^{\prime}$ Post-graduates, Institute of Ophthalmology, JNMCH, Aligarh Muslim University, Aligarh, India \\ ${ }^{2}$ Assistant Professor, Institute of Ophthalmology, JNMCH, Aligarh Muslim University, Aligarh, India
}

Article Info

\section{*Corresponding author: \\ Rahul Gupta \\ Institute of Ophthalmology \\ JNMCH, Aligarh Muslim University \\ Aligarh, India \\ E-mail: drrahulgupta06@gmail.com}

Received: February 13, 2016

Accepted: April 6, 2016

Published: April 11, 2016

Citation: Gupta R, Jahan S, Nagpal G, Akhtar $\mathrm{N}$, Waris A. Idiopathic Polypoidal Choroidal Vasculopathy - A Case Report. Madridge J Ophthalmol. 2016; 1(1): 1-4. doi: 10.18689/mjop-1000101

Copyright: @ 2016 The Author(s). This work is licensed under a Creative Commons Attribution 4.0 International License, which permits unrestricted use, distribution, and reproduction in any medium, provided the original work is properly cited.

Published by Madridge Publishers

\begin{abstract}
Idiopathic polypoidal choroidal vasculopathy (IPCV) is a peculiar form of choroidal neovascularization. The clinical feature of recurrent serous retinal leakage and retinal hemorrhage varies from a single lesion to multifocal, from self-limited to recurrent form.

Caucasian, African-American and Japanese patients with IPCV have been reported in the literature. However, research and case reports about IPCV in young Indian patients are relatively rare. Here, we present a case of IPCV in a young Indian female who presented to our out-patient department with diminution of vision
\end{abstract}

Keywords: Choroidal neovascularization; Idiopathic polypoidal choroidal vasculopathy; Retinal pigment epithelial detachment.

Abbreviations: IPCV: Idiopathic polypoidal choroidal vasculopathy; PED: Pigment epithelial detachments; VA: Visual acuity; ICG: Indocyanine green angiography; FFA: Fundus fluorescein angiography; PDT: Photodynamic therapy

\section{Introduction}

IPCV was first described by Yanuzzi in 1982 [1,2] in the macular disease society meeting as a disease occurring in females of African-American origin characterized by hemorrhagic lesion of the macula. This was also described as Posterior Uveal Bleeding syndrome [1].

Clinically, it is characterized by sero-sanguineous retinal pigment epithelial detachments in the posterior pole and can be single or multiple, persistent or regressed, recurrent or non-recurrent.

The disorder was poorly understood earlier and initially designated as recurrent retinal pigment epithelial detachments (PED) and posterior uveal bleeding syndrome [3-6]. However, the main pathology as described by Yanuzziet et al. $[1,3,7]$ involves the choroidal circulation, and the characteristic lesion is an inner choroidal vascular network of vessels ending in an aneurysmal bulge or an outward projection, visible clinically as a reddish orange spheroidal, polyp like structure [8]

\section{Case Report}

A22 year old female presented to Ophthalmology OPD of Institute of Ophthalmology, Jawahar Lal Nehru Medical College, Aligarh with chief complaints of diminution of vision in right eye for the past 3 months. 
The diminution of vision was painless, slow in onset, progressed for first 2 months and then was stationary for the past 1 month. There was no associated history of any redness, watering, discharge, photophobia, past fever, trauma, past surgical procedure or long term oral or ocular medication intake. There is no history of similar presentation in the past or any similar history in the immediate family. The systemic and local examination of the patient revealed no abnormality.

On ocular examination, the anterior segment was within normal limits. The Anterior Chamber was normal and there were no cells or flare. The uncorrected visual acuity in Right Eye was $6 / 60$ while that in left eye was $6 / 6$. Upon refraction, the best corrected visual acuity in right eye was $6 / 36$ with $-2 D$ spherical correction. It was $6 / 6$ in left eye. The Intra Ocular Pressure of the patient measured through non contact tonometer was $15 \mathrm{~mm}$ of $\mathrm{Hg}$ and $14 \mathrm{~mm}$ of $\mathrm{Hg}$ in right and left eye respectively.

Fundus examination of both eyes was done under full dilatation. Resolving macular haemorrhage was seen in right eye, rest everything was within normal limits. Fundus examination of left eye was within normal limits. The patient was then referred to Retina Clinic of the institute. OCT and FFA were advised and done.

Upon doing FFA, we observed presence of a group of polypoidal vessels in an avascular area in the macular region. A small vascular leak was also observed in the region of the polypoidal vessels. OCT showed an increase in thickness in the macular region.

ICGA is the investigation of choice for visualisation of the choroidal vasculature but it could not be done due to its unavailability in the Institute.

The diagnosis of Idiopatihic Polypoidal Choroidal Vasculopathy which had bled was made on the basis of fundus, OCT and FFA findings. Patient was advised intravitreal anti-VEGF in RE.

Intravitreal Bevacizumab $(1.25 \mathrm{mg} / 0.5 \mathrm{ml})$ was given in the Right Eye. One week post-op, there was no significant improvement in the visual function and the fundus appearance. The patient has been asked to review after one month.

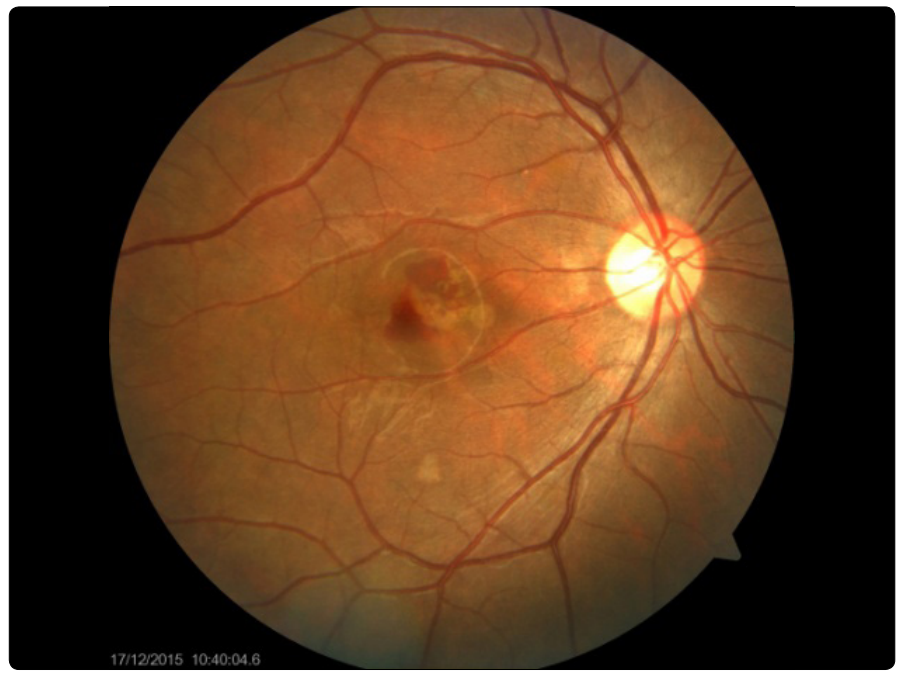

Figure 1: Fundus Photograph of the patient.

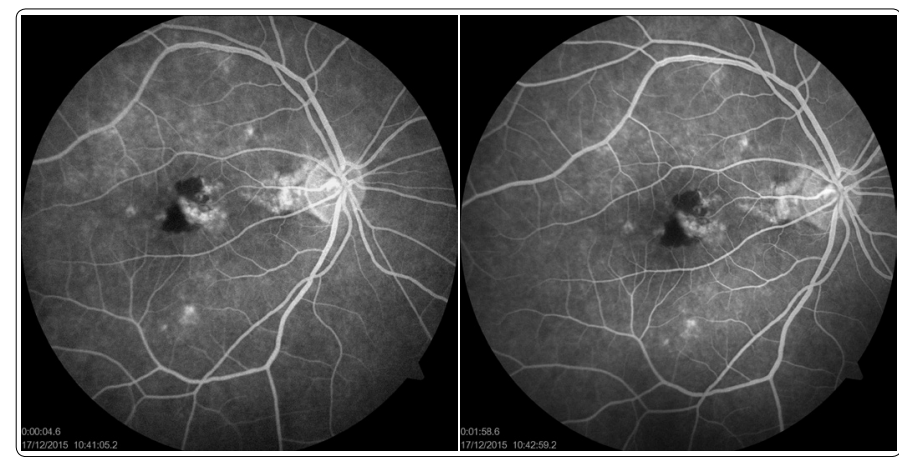

Figure 2: Fundus Photograph of the patient.

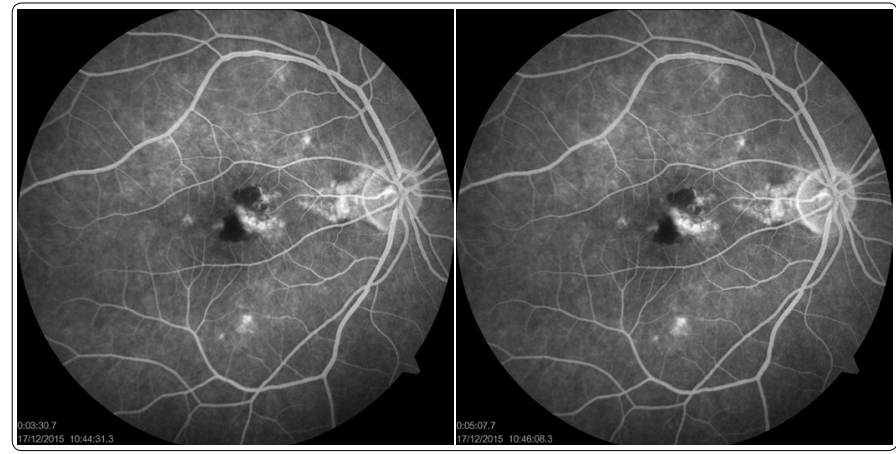

Figure 3: FFA images at different time intervals.

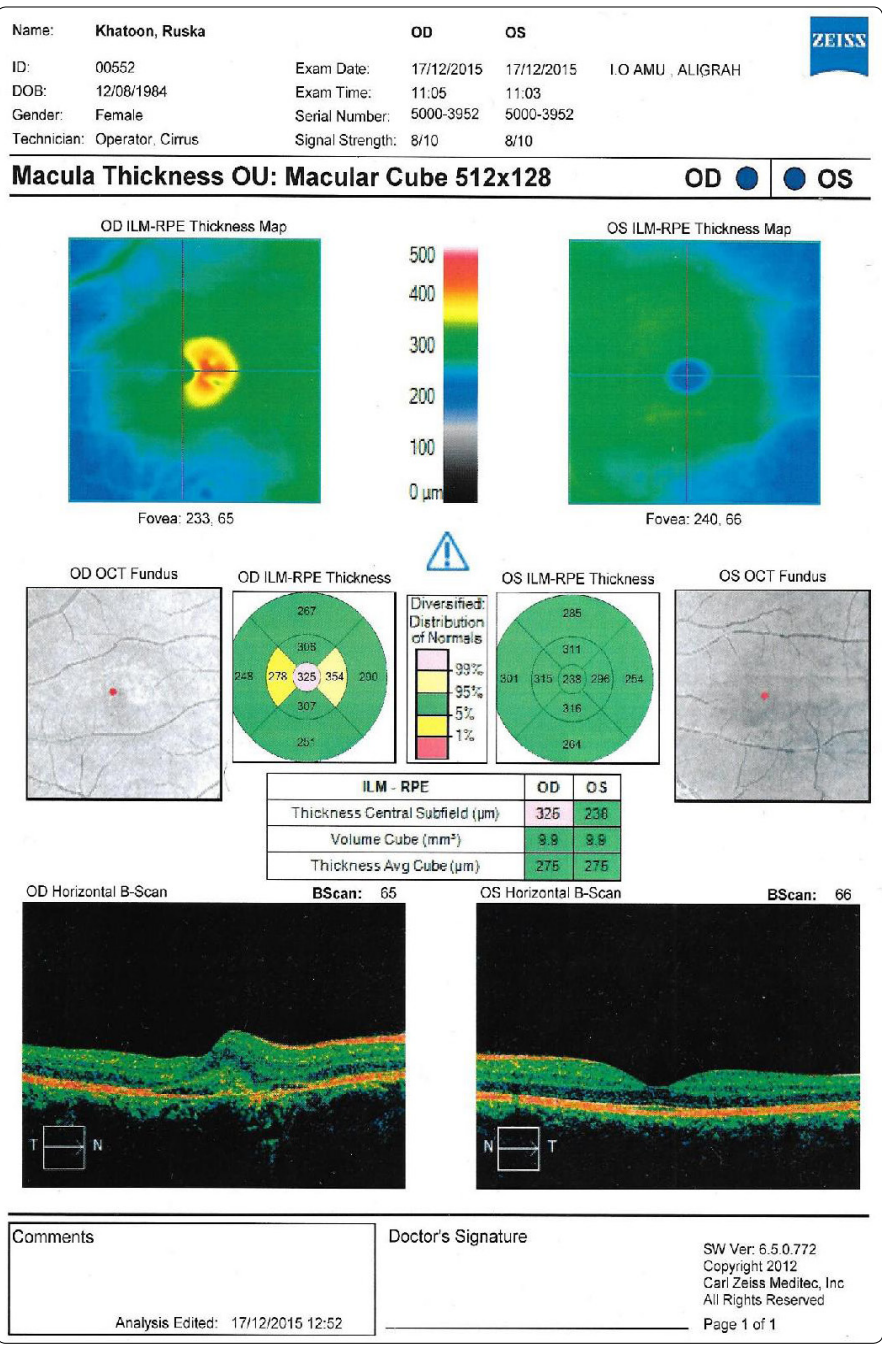

Figure 4: OCT of the patient. 


\section{Discussion}

IPCV is characterized by recurrent serous retinal leakage and hemorrhage in the elderly population, caused by vascular abnormalities in the inner choroid. This disease entity was first described by Yannuzzi in 1982 [1]. Kleiner and Brucker [9] later described the term posterior uveal bleeding syndrome as with multiple recurrent hemorrhage or serous fluid beneath the RPE and neurosensory retina.

\section{Demographics}

Several reports have suggested that the incidence of PCV is markedly high in black people, relatively high in Asian population and low in white people in contrast to age-related macular degeneration $[6,8,10]$. In blacks, PCV affects women more frequently and is usually associated with peripapillary involvement $[3-5,10,11]$. Several reports from the Asian population have shown a male preponderance, unilateral presentation with macular involvement $[6,7,10,11]$.

This disease is generally seen in the age group of 50-65 years. Caucasian subjects usually present at an older age. In the original reports in African-Americans, females were more often affected. However, in the reports from japan, male preponderance has been seen [6].

\section{Clinical Features}

Polypoidal choroidal vasculopathy more often presents as a bilateraldisease. The most common presenting symptom in PCV is decreased visual acuity (VA) due to sub-retinal fluid or sub-retinal hemorrhage.

It manifests as sub-retinal hemorrhage along with hemorrhagic pigment epithelial detachment. The polypoidal choroidal can be seen as orange lesions on biomicroscopy. This is generally more common if the lesions involve the larger choroidal vessels. Orange polyp like dilations are seen at the end of dilated choroidal vascular channels. Lesions associated with medium choroidal vessels need indocyanine green angiography for demonstration. The eye typically does not have any drusens unlike classical AMD. Also, there is no associated inflammation.

The classic presentation is sero-sanguinous detachment of neurosensory retina and retinal pigment epithelium. Fibrous proliferation as sequelae can occur but is much less common as compared to classical neovascularage related macular degeneration. Choroidal neovascular membrane can also occur secondarily. Rarely massive choroidal hemorrhage can occur and the prognosis in such cases has been uniformly poor.

Uyama et al. [12] described the natural course of the disease over a period of 2 years of observation. They described two different manifestations of the disease-the Exudative and the Hemorrhagic. The exudative variety is characterized by serous pigment epithelial detachment and secondary retinal detachment with little or no hemorrhage, while the hemorrhagic variety is characterized by variable amount of sub-retinal pigment epithelium hemorrhage and sub-retinal hemorrhage with or without breakthrough vitreous hemorrhage.

\section{Diagnostic Modalities}

PCV can be distinguished from AMD and CSR using indocyanine green angiography (ICG). Unfortunately, fundus fluorescein angiography (FFA), which is more readily available in clinical practices, often shows only non-specific PED and leakage that is present in a number of diseases $[13,14]$.

Indocyanine green angiography is the best test for delineating the lesions of PCV as it permits visualization of the choroids' vasculature and can show the typical aneurismal and spheroidal vascular dilation of the choroidal vessels [7]. Following characteristic changes are seen in different phases of ICG:

Initial phase: Initially the large choroidal vessels fill with the dye. The larger vessels of the PCV lesion fill before the retinal vessels. The polyps fill next.

Mid phase: Slow leakage takes place from the lesions that have filled up and the leakage spills over beyond the margins of the lesions.

Late phase: While the leak beyond the lesion remains, the lesion itself becomes hypo-fluorescent. However, small lesions may retain uniform staining.

Very late phases: Wash out phenomenon occurs with complete disappearance of the dye from lesions. However, the leaky (active) lesions retain the dye.

OCT: OCT can differentiate dome shaped PED from the nodular lesions characteristic of PCV. OCT may showpolyps under the RPE associated with serous RD. Generally the retina over the polyp is thickened.

\section{Management}

Visual prognosis of PCV is generally better as compared to $A M D$, mainly due to spontaneous regression and the containment of PCV lesions under the RPE preventing intrusion and leakage in the subretinal space.

A recent multicenter randomized controlled trial titled "EVEREST" (i.e. efficacy and safety of verteporfin photodynamic therapy in combination with ranibizumab or alone versus ranibizumab monotherapy in patients with symptomatic macular polypoidal choroidal vasculopathy) showed that treatment of PCV with a combination verteporfin photodynamic therapy (PDT) plus ranibizumab injections were well tolerated and highly effective in achieving complete regression of the polypoidal lesions in symptomatic PCV [6]. Specifically, combination PDT plus ranibizumab $0.5 \mathrm{mg}$ monthly injections $x 3$ showed complete polypoid regression in $77.8 \%$ of patients versus $71.4 \%$ for PDT alone and $28.6 \%$ for ranibizumab monotherapy [15]. Additionally, a long term 
visual outcome study of combination therapy showed that $88.2 \%$ of patients with PCV had either maintained or improved visual acuity 3 years after treatment [16]. In patients with incomplete regression of polypoidal lesions following initial combination treatment as assessed by ICG at 3 month followup, retreatment with PDT monotherapy or combination PDT/ ranibizumab is recommended $[13,15]$. If either FFA or ICG still detects leakage and continued signs of disease activity, retreatment with only ranibizumab is appropriate [13]. Although ranibizumab monotherapy is effective in reducing subretinal fluid, it is much less effective in achieving polyp regression. Intravitreal monotherapy may be chosen over PDT/ranibizumab combination therapy in patients who have good visual acuity, or who have any contraindications to PDT (e.g. abnormal liver function tests) [13].

Conflicts of Interest: The author(s) report(s) no conflicts of interest.

Consent: Patient has provided written consent for publication of this study.

\section{References}

1. Yannuzzi LA. Idiopathic polypoidal choroidal vasculopathy. Presented at: Macula Society Meeting, February 5, 1982, Miami, FL, USA.

2. Yannuzzi LA, Sorenson J, Spaide RF, Lipson B. Idiopathic polypoidal choroidal vasculopathy (IPCV). Retina. 1990; 10: 1-8.

3. Yannuzzi LA, Ciardella A, Spaide RF, Rabb M, Freund KB, Orlock DA. The expanding clinical spectrum of idiopathic polypoidal choroidal vasculopathy. Arch Ophthalmol. 1997; 115:478-85. doi: 10.1001/archopht.1997.01100150480005.

4. Stern RM, Zakov ZN, Zegarra H, Gutman FA. Multiple recurrent serosanguineous retinal pigment epithelial detachments in black women. Am J Ophthalmol. 1985; 100: 560-569.

5. Kleiner RC, Brucker AJ, Johnston RL. The posterior uveal bleeding syndrome. Retina. 1990; 10: 9-17.
6. Sho K, Takahashi K, Yamada H, et al. Polypoidal choroidal vasculopathy: incidence, demographic features, and clinical characteristics. Arch Ophthalmol. 2003; 121: 1392-1396. doi: 10.1001/archopht.121.10.1392

7. Spaide RF, Yannuzzi LA, Slakter JS, Sorenson J, Orlach DA. Indocyanine green video angiography of idiopathic polypoidal choroidal vasculopathy. Retina. 1995; 15: 100-110.

8. Moorthy RS, Lyon AT, Rabb MF, Spaide RF, Yannuzzi LA, Jampol LM Idiopathic polypoidal choroidal vasculopathy of the macula. Ophthalmology. 1998; 105: 1380-1385. doi: 10.1016/S0161-6420(98)98016-2

9. Kleiner RC, Brucker AJ, Johnston RL. Posterior uveal bleeding syndrome. Ophthalmology. 1984; 91: 110.

10. Ciardella AP, Donsoff IM, Huang SJ, Costa DL, Yannuzzi LA. Polypoidal choroidal vasculopathy. Surv Ophthalmol. 2004; 49: 25-37. doi: 10.1016/j. survophthal.2003.10.007

11. Gomi F, Tano Y. Polypoidal choroidal vasculopathy and treatments. Curr Opin Ophthalmol.2008; 19:208-212. doi:10.1097/ICU.0b013e3282fb7c33

12. Uyama $M$, Matsubara $T$, Fukushima I, et al. Idiopathic polypoidal choroidal vasculopathy in Japanese patients. Arch Ophthalmol. 1999; 117: 1035-1042. doi: 10.1001/archopht.117.8.1035

13. Koh AH, Expert PCV Panel, Chen LJ, et al. Polypoidal choroidal vasculopathy: evidence-based guidelines for clinical diagnosis and treatment. Retina. 2013; 33(4): 686-716. doi: 10.1097/IAE.0b013e3182852446

14. Honda S, Matsumiya W, Negi A. Polypoidal choroidal vasculopathy: clinical features and genetic predisposition. Ophthalmologica. 2014 231(2): 59-74. doi: $10.1159 / 000355488$

15. Koh AH, Lee WK, Chen LJ, et al. EVEREST Study: Efficacy and safety of verteporfin photodynamic therapy in combination with ranibizumab or alone versus ranibizumab monotherapy in patients with symptomatic macular polypoidal choridal vasculopathy. Retina. 2012; 32: 1453-1464. doi: 10.1097/IAE.0b013e31824f91e8

16. Kang HM, Koh HJ, Lee CS, Lee SC. Combined photodynamic therapy with intravitreal bevacizumab injections for polypoidal choroidal vasculopathy: long-term visual outcome. Am J Ophthalmol. 2014; 157(3): 598-606. doi: 10.1016/j.ajo.2013.11.015 